\title{
Heterogeneity of deficits in developmental dyslexia and implications for methodology
}

\author{
RANDI C. MARTIN \\ Rice University, Houston, Texas
}

\begin{abstract}
Most of the studies overviewed by Farmer and Klein (1995) in their review of temporal processing disorders in developmental dyslexia have taken a group study approach in which a group of dyslexic readers is compared with a group of normal readers on some task thought to be relevant to the reading disorder. Because of the acknowledged heterogeneity of deficits among developmental dyslexics, this group study methodology is inappropriate and is likely to lead to findings in one lab that cannot be replicated in another. The single case study methodology, which has been used successfully in the study of adult neuropsychological impairments, should be adopted in the study of developmental impairments as well. In the case study approach, each individual is studied thoroughly with tasks designed to tap the various components of the cognitive domain under study in order to determine which components are spared and which impaired. Data are not averaged, but reported separately for each case. Some recent findings from case studies on developmental dyslexia are reviewed and suggestions are made as to how the case study approach could be used in analyzing whether a temporal processing disorder, or any other hypothesized factor, is causal to the reading disorder.
\end{abstract}

In many respects Farmer and Klein (1995) have done an admirable job of summarizing and evaluating a large body of evidence on whether groups of dyslexic readers differ from groups of normal readers on various aspects of temporal processing tasks in the auditory and visual modalities. Their review establishes that at least some subset of dyslexic readers perform poorly on such tasks. They have presented data and arguments that provide a plausible case as to how a temporal processing deficit might be related to a deficit in processing speech. That is, given the rapidity of change in the speech signal over time, a temporal processing disorder might lead to impaired development of phonological representations. Thus, they have provided a means of linking a temporal processing disorder in the auditory domain to the phonological deficits that have been noted in many dyslexic readers. As has been argued by several researchers, a phonological deficit could prevent the development of letter-sound conversion skills and consequently impede the development of reading (e.g., Bradley \& Bryant, 1983; Stanovich, 1988). (Farmer and Klein's efforts at linking a temporal processing disorder in the visual domain to possible causes of dyslexia seem less convinc-

Preparation of this manuscript was supported by NIH-NIDCD Grant 00218 to Rice University. The author would like to thank Mary Lesch and Henry L. Roediger III for their comments on an earlier version. Correspondence should be addressed to R. C. Martin, Department of Psychology, Rice University, P.O. Box 1892, Houston, TX 77251 (e-mail: rmartin@rice.edu). ing; see the comment by Rayner, Pollatsek, \& Bilsky, 1995, for discussion.) The overview that Farmer and Klein have provided will most likely serve the important role of stimulating research on whether the phonological deficits noted in many dyslexic readers might be caused by a temporal processing disorder.

In my objection to Farmer and Klein's paper, I am concerned not so much with the specifics of their arguments, as with the general approach to the study of developmental dyslexia exemplified in the studies contained in their review. Thus, my criticisms are not directed so much at the analyses and interpretations offered by Farmer and Klein, but rather at the previous studies which form the basis of their interpretations. Although Farmer and Klein ackowledge that there may be several different sources of developmental dyslexia, in most of the studies that they review, the researchers have treated developmental dyslexic subjects as a homogeneous group and seem to have sought a single underlying cause of the deficit. Indeed, the history of the field of developmental dyslexia has been a sequence of different unitary explanations (e.g., unusual distribution of capacities across the two hemispheres, visual perceptual deficits, impairments in the control of eye movements, phonological deficit, temporal processing deficit), with the proponents of one unitary hypothesis seeking to discredit the rest (see Ellis, 1985, for a review). As Ellis notes, a disease model seems to be at work here, in which dyslexia is treated as a disease such as tuberculosis, which one either has or does not, and it is the researchers' task to discover the 
one cause of this disease. However, dyslexia is more analogous to a symptom, such as a cough, than to a disease. Just as a cough can result from many different disease processes, dyslexia could result from different underlying sources. Given the complexity of reading single words and text and the variety of perceptual and cognitive processes involved (i.e., visual, orthographic, phonological, attentional, semantic, syntactic, memorial), there would seem to be no a priori reason why only one of these should fail to develop normally nor, alternatively, why there should be only one underlying component that fails to develop normally and causes the downfall of all.

A corollary of the single-deficit approach is the use of group study methodology, employed by most of the studies reported in the Farmer and Klein paper. That is, a group of dyslexic individuals (defined as having poor reading abilities relative to other cognitive skills) is compared with a control group on some measure thought to be related to the reading disorder. This type of group study methodology was common in studies of adult neuropsychological disorders in the past, but it has come under increasing attack in recent years (Caramazza, 1984; Caramazza \& McCloskey, 1988; Ellis, 1987). The basic problem with the group study approach is that it is theoretically unjustifiable to average the performance of individuals who have different functional deficits. In the worst case, the average performance may characterize none of the individual subjects' performance. In the best case, the average performance may reflect a majority of cases, but hide the existence of divergences from the average in a significant number of cases. Unfortunately, when a statistically significant difference is obtained between the disordered groups' performance and that of the control subjects, the inference often seems to be drawn that the difference characterizes most, if not all, of the individuals in the disordered group. In fact, the distributions might be nearly entirely overlapping but with small differences between the group means that are significant with large enough sample sizes. ${ }^{1}$ For example, as is indicated in Farmer and Klein's paper, in one of the earlier studies on a possible temporal processing disorder in disabled readers, Tallal (1980) reported that 12 of 20 disabled readers scored within normal range on the temporal processing task even though a significant group difference was obtained. Thus, it is unlikely that a temporal processing deficit was the source of the reading difficulties for the majority of the dyslexic subjects in that study. However, the results of that study are typically cited as showing that dyslexic readers have a temporal processing deficit.

In commenting on the variability of the reading-disabled group on the temporal processing task, Tallal (1980) stated that "we may be able to learn as much about reading disabilities by looking at different patterns of performance within groups of reading-impaired children as we can by looking at differences between reading-impaired children and normal children" (p. 195). However, this suggestion seems not to have been taken to heart by most who have followed up on her findings. Instead, researchers inves- tigating temporal processing disorders have continued to treat developmental dyslexics as a single group. A discouraging, but predictable, outcome of group study methodology applied to groups composed of individuals with differing underlying deficits is nonreplicability. That is, the findings in one lab cannot be replicated in another, because the relative proportion of individuals with a particular deficit differs across the samples obtained by different researchers. Examples of contradictory findings are evident in the studies presented in Farmer and Klein's review. For example, the study by Shapiro, Ogden, and Lind-Blad (1990) showed that dyslexics could read all words as well as could controls when given sufficiently long exposure durations. Along similar lines, the Hill and Lovegrove (1992) study did not find an impairment in the reading of singly presented words among the dyslexic subjects. These findings contradict many other studies in the literature which show that dyslexic children make errors in reading short words even with unlimited exposure duration (see, e.g., the Castles \& Coltheart, 1993, study cited in Farmer \& Klein; all of the individual case studies discussed below in this comment; and, in addition, the following: Aaron, Olsen, \& Baker, 1985; Coltheart, Masterson, Byng, Prior, \& Riddoch, 1983; Seymour \& MacGregor, 1984).

Faced with nonreplications of their work, the usual response of researchers is to question details of the other lab's methodology or question whether the other lab's subjects were really dyslexic, and to hang on to their own favorite explanation for the cause of dyslexia. Such failures to replicate have been common in the field of dyslexia research, as have been acrimonious debates between advocates of differing underlying causes. It should be noted, however, that some of the researchers who use a group study approach have acknowledged the heterogeneity of deficits among dyslexic readers and have tried to remedy the situation by attempting to classify dyslexic readers into a small number of homogeneous subgroups (Boder, 1973; Doehring, Trites, Patel, \& Fiedorowicz, 1981). As Farmer and Klein imply, such classification schemes have not been notably successful, because of the difficulties in determining how many such subtypes there should be and in determining the subtype to which a particular reader belongs. However, given the number of cognitive components involved in reading and the possibility of continuous variation in the degree of impairment in a particular component, there is no reason to suspect that dyslexics will fall into a small number of clearly identifiable subtypes. That is, any given dyslexic individual might be developmentally impaired on one or several components, and the degree of impairment might differ across components. If so, it is unlikely that a simple typology can be developed that will allow researchers to readily classify most cases. However, analysis of variation of deficits among dyslexic readers does not have to be postponed until a typology can be established.

The alternative to the group study (whether of a single group or of a set of subtypes) is the case study approach, a methodology which has proved successful in the study 
of adult neuropsychological disorders (see Ellis \& Young, 1988, and Shallice, 1988, for an overview of findings from studies taking this approach). In this approach, individual cases are studied in detail with respect to a cognitive model of the affected processing domain (e.g., object recognition, language production, memory). The goal of this enterprise is to use the model to determine cognitive components that are spared or impaired in an individual case. Reciprocally, the pattern of deficits across one or several cases (studied individually) can be used to determine whether the existing model is sufficient to explain the observed patterns of performance or whether modifications are needed. The study of acquired dyslexia (i.e., impaired reading resulting from brain damage due to, for example, a stroke or head injury) has been one area in which this approach has resulted in significant advances in the understanding of the component processes involved in reading and their disruption with brain damage.

Figure 1 presents a dual-route route model of reading which has been developed on the basis of studies of normal subjects and case studies of acquired dyslexia. Even in this model, which does not attempt to account for reading beyond the single word, a number of components and pathways are involved. Patterns of reading performance and correlated performance on other types of tasks have been related to disruptions in the representations or pathways in the model. The two routes in this model are a sublexical route and a lexical route. The sublexical route involves the conversion of letters or letter

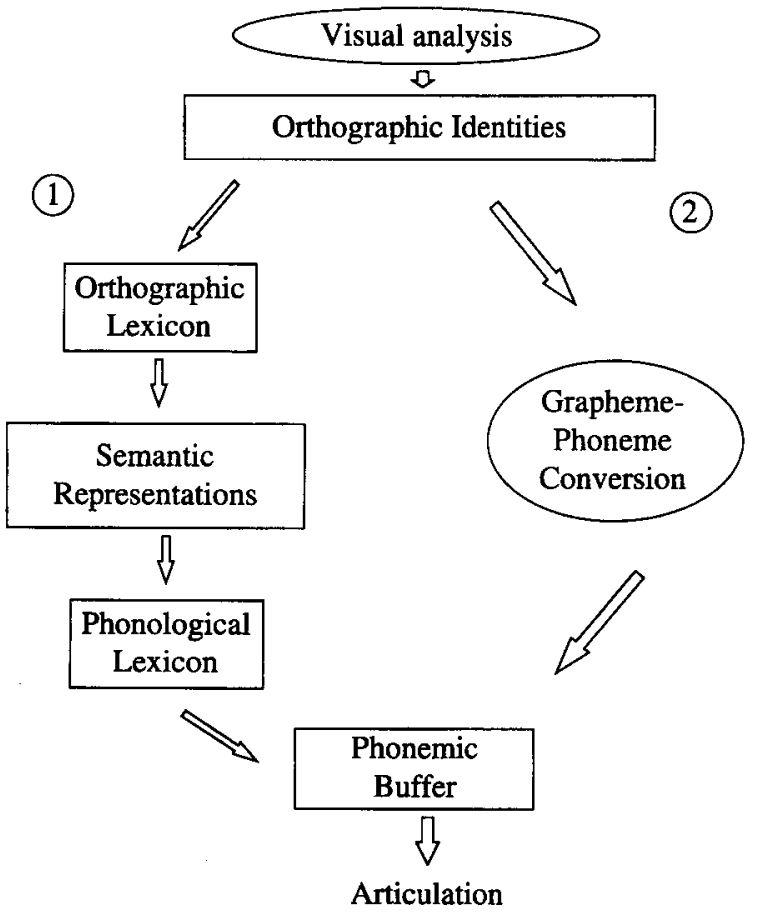

Figure 1. A dual-route model of reading commonly employed in studies of acquired dyslexia showing the (1) lexical route and (2) sublexical route. groups into sounds (i.e., grapheme-phoneme conversion). Novel letter strings (i.e., pronounceable nonwords such as blim) and words with regular letter-sound correspondences (e.g., gift, ship) may be read by this route. The lexical route involves mapping letter identities of a word onto a whole-word (i.e., lexical) orthographic representation. The lexical orthographic representation provides access to the word's semantic representation, which, in turn, provides access to the word's phonological representation. ${ }^{2}$ Familiar words with either regular (e.g., gift) or irregular (e.g., sword, pint) letter-sound correspondences may be read by this route. Acquired dyslexia cases have been reported that show a disruption of the sublexical route but a preservation of the lexical route, demonstrated by an inability to read nonwords but an ability to read words (see, e.g., Beauvois \& Derousne, 1979; Funnell, 1983). The reverse dissociation has also been reported in cases that have a disruption of the lexical route but a preservation of the sublexical route, demonstrated by a disrupted ability to read irregular words, but a good ability to read regular words and nonwords (see, e.g., Bub, Cancelliere, \& Kertesz, 1985; McCarthy \& Warrington, 1986). ${ }^{3}$

In addition to these contrasting dissociations between disruptions of the sublexical and lexical routes, other distinctions can be made among cases of acquired dyslexia. In several patients, the deficit appears to occur in a visual analysis stage that would be a prerequisite to processing in either route (see, e.g., Ellis, Flude, \& Young, 1987; Shallice \& Warrington, 1977). Among patients who have a deficit in the lexical route, the disruption may occur along any point in the pathway from wholeword orthographic representations to pronunciations. Cases have been documented with the following deficits in components: (1) a disruption of lexical orthographic representations (Behrmann \& Bub, 1992), (2) a disruption of semantic representations (Patterson \& Hodges, 1992), and (3) a deficit in accessing the phonological lexicon from semantics (Howard \& Franklin, 1988). Similarly, patients showing a disruption in the sublexical route may have different deficits. For example, some patients may have a disrupted knowledge of grapheme-phoneme conversion (Funnell, 1983), whereas others may retain such knowledge but have difficulty maintaining all of a nonword's phonemes in a short-term memory buffer prior to blending these into a pronunciation of the letter string as a whole (Caramazza, Miceli, \& Villa, 1986).

The success of the cognitively based case study approach in neurospychology has led to the application of the dual-route model to the analysis of cases of developmental dyslexia (see, e.g., Castles \& Coltheart, 1993; Temple \& Marshall, 1983). Some have questioned the appropriateness of the direct transfer of an adult model of reading to the study of reading development and its disorders (Hulme \& Snowling, 1992). For example, some have suggested that the development of the sublexical route is a necessary prerequisite to the development of a lexical route (Frith, 1985), and, consequently, one would 
not expect to see developmental dyslexics who have a normally functioning lexical route together with a deficient sublexical route (but see Temple, Jeeves, \& Vilarroya, 1990, and Stothard, Snowling, \& Hulme, in press, for counterexamples). Nonetheless, such case studies have provided strong evidence of the variability in patterns of performance in cases of developmental dyslexia. For the most part, these case studies were not covered in Farmer and Klein's review, which is justifiable, since these studies have not dealt directly with the issue of temporal processing. However, some of the patterns of reading difficulties uncovered in these studies would seem to be difficult to relate to a temporal processing disorder. A few such studies will be briefly discussed here to illustrate this point. Although Farmer and Klein indicate that a temporal processing disorder is unlikely to be the cause of all cases of developmental dyslexia, there is little discussion of the data which already support this conclusion. The studies cited below should direct the reader to some relevant literature.

Broom and Doctor (1995) presented the case of a child, D.F., for whom it appeared that the lexical route had failed to develop normally. D.F. was able to read regular words and nonwords at the level of reading-agematched controls, but performed significantly poorer than these controls on reading irregular words. In addition, his spelling was poor, and he often produced phonologically plausible, but incorrect, spellings (e.g., writing gard for guard). It should be noted that correct reading of regular words and nonwords implies accurate letter perception and accurate grapheme-phoneme conversion and sound-blending abilities. It might be thought that such an over-reliance on grapheme-phoneme conversion would be rare in developmental dyslexia, given the phonological deficits noted in many such cases. However, Castles and Coltheart (1993), mentioned in Farmer and Klein's review, found that 10 of the 56 dyslexic subjects who were studied performed like D.F., reading nonwords at a normal level but reading irregular words at a subnormal level. Another 6 who were impaired relative to controls on both nonword and irregular word reading nevertheless showed substantially more impaired irregular word reading than nonword reading. A phonological deficit hypothesis or a rapid temporal processing deficit would seem to be hard pressed to account for a normal ability to carry out grapheme-phoneme conversion but an impaired ability to develop whole-word orthographic representations. ${ }^{4}$ Also, a fairly large subgroup ( 29 out of 56 ) of these subjects showed evidence of a disruption in letter-sound conversion, reading regular and irregular words at about the same level, and performing very poorly on reading nonwords, which would be consistent with the phonemic deficit hypothesis (and also with the temporal processing deficit account). If only group data analyses had been performed, the existence of the subjects with well-developed letter-sound conversion abilities would have been missed.

As in the case of adult acquired dyslexia, even among developmental dyslexic subjects who show impairment of phonological processes (and consequent difficulty in reading nonwords), there is evidence that a fine-grained analysis reveals differences in the specific component that has failed to develop normally. For example, one dyslexic subject had normal speech perception but a poor ability to read nonwords and very poor phonological short-term memory. Presumably, the poor short-term memory impaired her ability to maintain and combine all the phonemes in a letter string, resulting in poor reading of nonwords (Campbell \& Butterworth, 1985). Other cases with poor nonword reading abilities have been reported who do show deficits in speech perception (e.g., T.W. in Snowling, Stackhouse, \& Rack, 1986), which could plausibly affect their ability to develop accurate phonological representations for words, and consequently impair phonological analysis involved in learning lettersound correspondences. Snowling and Hulme (1989) reported a developmental case whose phonological deficits appeared mainly on tests involving speech production (e.g., naming and repetition), but who showed good speech perception. It is difficult to see how all these different types of phonological deficits could be related to a temporal processing disorder.

Other quite different patterns of reading disruption have also been reported which appear unrelated to phonological coding at the sublexical or lexical level. For example, the adult developmental dyslexic reported by Rayner, Murphy, Henderson, and Pollatsek (1989) read single words at a normal level but experienced considerable difficulty with text. This subject showed evidence of a visual attentional deficit affecting his reading, which was not demonstrated by two other dyslexic subjects included in this study.

Thus, the case study approach has revealed a number of differing patterns of deficits on reading tasks and on other tasks theoretically related to components of the reading process. On the face of it, many of the patterns of deficits would seem unlikely to be related to a temporal processing disorder. Although Farmer and Klein suggest that factors other than temporal processing disorders may be causally related to developmental dyslexia, they seem to hold out the possibility that temporal processing disorders may underlie a large proportion of cases; however, they suggest that these temporal processing disorders may ameliorate over time (particularly in the visual modality) and may do so at different rates for different individuals. The result is a plethora of different patterns of reading impairment among developmental dyslexics. As Farmer and Klein acknowledge, the evidence is mixed that temporal processing disorders may resolve at any earlier age in the visual modality than in the auditory modality, given that in some of the studies that they cite, continuing visual temporal processing deficits were found in older dyslexic subjects (e.g., Brannan \& Williams, 1988; May, Williams, \& Dunlap, 1988). The temporal processing hypothesis as stated seems so vague and flexible that any pattern of reading deficit could be attributed to some combination of existing and prior temporal processing deficits in the audi- 
tory and visual modalities. As Farmer and Klein acknowledge, longitudinal data are required in order to document that temporal processing difficulties did exist at some point although they may have disappeared later. Given that no such longitudinal studies have been carried out, the collection of such data would seem to be a necessary step in order to address Farmer and Klein's hypothesis about amelioration over time. However, in order to make the temporal processing hypothesis falsifiable, it would be necessary to have some more precise predictions about what the consequences of amelioration of a temporal processing disorder in one modality should be for given stages in development, and to test these hypotheses on an individual case basis.

Despite their repeated acknowledgment of the heterogeneity of deficits across cases, Farmer and Klein seem reluctant to endorse the case study approach (p. 476). They argue that the case study approach provides no information on the relative frequencies of different patterns of deficits. However, taking a case study approach does not mean that one must examine only one child at a time. A large sample of children may be studied simultaneously. The only requisite is that each of the children be studied thoroughly, with tasks designed to tap different components of the reading process, and that the data from each child be presented separately, rather than averaged. In fact, the Castles and Coltheart (1993) study that they mention is an example of the case study approach applied to 56 children simultaneously. Farmer and Klein also point out that heterogeneity of deficits is further complicated by possible changes in these deficits over time (p. 476). Consequently, longitudinal studies would seem to be required. The advantages of a case study approach would seem to be particularly evident in a longitudinal study where the dangers of averaging would be compounded, given that the rate of change or the change in pattern of performance might differ widely across individuals. In fact, a few longitudinal studies of developmental dyslexia cases have already been carried out (Case J.M.-Hulme \& Snowling, 1992; Snowling \& Hulme, 1989; Case L.F.-Stothard et al., in press), demonstrating the feasability of the approach. Of course, having to test several children on a large number of tasks, perform a cognitive analysis of the patterns of deficits for each, and follow possible changes in these patterns over several years may seem a daunting prospect to the researcher. Nonetheless, there would seem to be no alternative, if one is to take seriously the notion that there may be different underlying causes of dyslexia in different children.

The final section of Farmer and Klein's paper deals with the question of how one might establish a causal link between a temporal processing disorder and a reading disorder. Their overview establishes that some subset of individuals with developmental dyslexia perform more poorly than controls on individuation tasks (i.e., tasks which determine the interstimulus interval at which two stimuli rather than one continuous or fused stimulus is perceived) and on temporal order judgment tasks (i.e., tasks requiring the recognition of sequences of brief stimuli). As they point out, it would be useful to know what proportion of individuals with a phonological processing deficit have difficulties with rapid temporal processing in the auditory modality, and to know whether auditory temporal processing deficits in young children predict later phonological deficits. However, such data, while necessary for establishing the plausibility of a temporal processing deficit as a contributing factor, would not go very far in distinguishing correlation from causation. As Ellis (1985) suggested, it is possible that dyslexia results from a disorder in the development of the left hemisphere. Such a left-hemisphere disorder might cause both phonological deficits and deficits in processing rapidly presented nonspeech auditory stimuli, although different brain areas subserve the two functions. The greater the left-hemisphere abnormality, the more likely both will be affected. Consequently correlations between degree of temporal processing deficit and degree of phonological deficit would not necessarily indicate that one causes the other. ${ }^{5}$ In order to establish that an auditory temporal processing deficit is causally related to a phonological deficit, it would be necessary to become more precise about what implications a temporal processing disorder should have for phonological processing. Given that dissociable deficits in speech perception, short-term memory, and speech production have been documented in developmental dyslexia, which of these would plausibly be related to a temporal processing deficit? Depending on how a temporal processing deficit is defined, it would seem that some quite specific predictions could be made. For example, if a temporal processing deficit is taken to mean a deficit recognizing stimuli for which the spectral information changes rapidly over time (see Studdert-Kennedy \& Mody, 1995 , for a discussion of the ambiguity on this point), this deficit can explain the greater impairment for perceiving sequences of stop-vowel syllables than sequences of vowels that has been reported in some dyslexic readers (e.g., Reed, 1989). Consequently, one might expect that for individual dyslexic readers who show a temporal processing disorder for rapidly changing nonspeech stimuli, worse perception of stops than of vowels should be found, and moreover, letter-sounding abilities should also reflect worse performance with stops than vowels. If such fine-grained correspondences could be noted (on a case-by-case basis), more compelling evidence for the causal nature of the temporal processing deficit would be obtained. Of course, the attribution of causation to the temporal processing disorder for even such a pattern of deficits would be undermined if cases were found in which these symptoms were dissociated: (1) a temporal processing disorder for nonspeech auditory stimuli, but no greater difficulty with stops than with vowels in perception or letter sounding, or (2) greater difficulty with stops than vowels, but no temporal processing disorder for nonspeech stimuli. Cases of the first type would be more damaging to the claim that a temporal processing disorder underlies any case of developmental dyslexia, 
whereas cases of the second type might be accounted for on the grounds that some developmental dyslexics have a phonological deficit independent of a temporal processing disorder.

To summarize, the group study approach has dominated the study of developmental dyslexia, with some unfortunate consequences. A number of lines of evidence suggest that different patterns of reading deficits are observed across different dyslexic individuals, making it unlikely that any single causal factor underlies all cases. Future work aimed at determining the causal factors underlying developmental dyslexia will have to take the heterogeneity of deficits seriously. The argument presented here is that such work will have to take a case study approach. A few case studies have already been carried out that have uncovered interesting patterns of deficits, some of which seem difficult to account for in terms of a temporal processing disorder. Whether a temporal processing disorder, or any other factor, can be seen as causally related to any cases of developmental dyslexia will depend on future research which examines, on a case-by-case basis, the extent to which this hypothesized factor is necessarily associated with specific patterns of reading deficits.

\section{REFERENCES}

Aaron, P. G., Olsen, J., \& Baker, C. (1985). The dyslexic college student: Is he also dysphasic? Cognitive Neuropsychology, 2, 115-147.

Beauvois, M.-F., \& Derousne, J. (1979). Phonological alexia: Three dissociations. Journal of Neurology, Neurosurgery \& Psychiatry, 42, 1115-1124.

Behrmann, M., \& Bub, D. (1992). Surface dyslexia and dysgraphia: Dual routes, single lexicon. Cognitive Neuropsychology, 9, 209-25I

BODER, E. (1973). Developmental dyslexia: A diagnostic approach based on three atypical reading-spelling patterns. Developmental Medicine \& Child Neurology, 15, 663-687.

Bradley, L., \& Bryant, P. E. (1983). Categorizing sounds and learning to read-a causal connection. Nature, 301, 419-421.

Brannan, J. R., \& Williams, M. C. (1988). Developmental versus sensory deficit effects on perceptual processing in the reading disabled. Perception \& Psychophysics, 44, 437-444.

Broom, Y. M., \& Doctor, E. A. (1995). Developmental surface dyslexia: A case study of the efficacy of a remediation programme. Cognitive Neuropsychology, 12, 69-100.

Bub, D., Cancelliere, A., \& Kertesz, A. (1985). Whole-word and analytic translation of spelling to sound in a non-semantic reader. In K. E. Patterson, J. C. Marshall, \& M. Coltheart (Eds.), Surface dyslexia: Neuropsychological and cognitive studies of phonological reading ( $\mathrm{pp} .15-34$ ). London: Erlbaum.

Camprell, R., \& Butterworth, B. (1985). Phonological dyslexia and dysgraphia in a highly literate subject: A developmental case with associated deficits of phonemic processing and awareness. Quarterly Journal of Experimental Psychology, 37A, 435-475.

Caramazza, A. (1984). The logic of neuropsychological research and the problem of patient classification. Brain \& Language, 21, 9-20.

Caramazza, A., \& McCloskey, M. (1988). The case for singlepatient studies. Cognitive Neuropsychology, 5, 517-528.

Caramazza, A., Miceli, G., \& Villa, G. (1986). The role of the (output) phonological buffer in reading, writing and repetition. Cognitive Neuropsychology, 3, 37-76.

CAstles, A., \& Coltheart, M. (1993). Varieties of developmental dyslexia. Cognition, 47, 149-180.

Coltheart, M., Curtis, B., Atkins, P., \& Haller, M. (1993). Models of reading aloud: Dual-route and parallel-distributed processing approaches. Psychological Review, 100, 589-608.
Coltheart, M., Masterson, J., Byng, S., Prior, M., \& Riddoch, J. (1983). Surface dyslexia. Quarterly Journal of Experimental Psychology, 37A, 468-495

Doehring, D. G., Trites, R. L., Patel, P. G., \& Fiedorowicz, C. A. M. (1981). Reading disabilities. New York: Academic Press.

ELLIS, A. (1985). The cognitive neuropsychology of developmental (and acquired) dyslexia: A critical survey. Cognitive Neuropsychology, 2, 169-205.

ELLIs, A. (1987). Intimations of modularity, or, the modularity of mind. In M. Coltheart, G. Sartori, \& R. Job, The cognitive neurospychology of language (pp. 397-408). Hove, U.K.: Erlbaum.

Ellis, A., Flude, B., \& Young, A. (1987). "Neglect dyslexia" and the early visual processing of letters. Cognitive Neuropsychology, 4, 439-464.

Ellis, A., \& Young, A. (1988). Human cognitive neuropsychology. Hove, U.K.: Erlbaum.

FARMER, M. E., \& KLEIN, R. (1995). The evidence for a temporal processing deficit linked to dyslexia: A review. Psychonomic Bulletin \& Review, 2, 460-493.

FRITH, U. (1985). Beneath the surface of developmental dyslexia. In K. E. Patterson, J. C. Marshall, \& M. Coltheart (Eds.), Surface dyslexia (pp. 301-330). London: Routledge \& Kegan Paul.

FUNNELL, E. (1983). Phonological processes in reading: New evidence from acquired dyslexia. British Journal of Psychology, 74, 159-180.

Goodall, W. C., \& Phillips, W. A. (1995). Three routes from print to sound: Evidence from a case of acquired dyslexia. Cognitive Neuropsychology, 12, 113-148.

Hill, R., \& Lovegrove, W. (1992). One word at a time: A solution to the visual deficits in SRDs? In S. F. Wright, R. Groner, \& R. Kaufmann-Hayoz (Eds.), Visual aspects of dyslexia and its remediation (pp. 65-76). Amsterdam: Elsevier, North-Holland.

Hillis, A., \& Caramazza, A. (1991). Mechanisms for activating lexical representations for output: Evidence from a category-specific semantic deficit. Brain \& Language, 40, 106-144.

Howard, D., \& FrankLin, S. (1988). Missing the meaning? A cognitive neuropsychological study of processing of words by an aphasic patient. Cambridge, MA: MIT Press.

Hulme, C., \& SNowling, M. (1992). Deficits in output phonology: An explanation of reading failure? Cognitive Neuropsychology, 9, 4772 .

Ludlow, C. L., Cudahy, E. A., Bassich, C., \& Brown, G. L. (1983). Auditory processing skills of hyperactive, language-impaired and reading-disabled boys. In E. Z. Lasky \& J. Katz (Eds.), Central auditory processing disorders (pp. 163-184). Baltimore: University Park Press.

May, J. G., Williams, M. C., \& DunlaP, W. P. (1988). Temporal order judgements in good and poor readers. Neuropsychologia, 26, 917-924.

McCarthy, R., \& Warrington, E. K. (1986). Phonological reading: Phenomena and paradoxes. Cortex, 22, 359-380.

MCCloskey, M. (1993). Theory and evidence in cognitive neuropsychology: A radical response to Robertson, Knight, Rafal, and Shimamura (1993). Journal of Experimental Psychology: Learning, Memory, \& Cognition, 19, 718-734.

PatTERson, K. (1990). Alexia and neural nets. Japanese Journal of Neuropsychology, 6, 90-99.

Patterson, K., \& Hodges, J. (1992). Deterioration of word meaning: Implications for reading. Neuropsychologia, 30, 1025-1040.

Patterson, K., Seidenberg, M., \& McClelland, M. (1989). Connections and disconnections: Acquired dyslexia in a computational model of reading processes. In R. G. M. Morris (Ed.), Parallel distributed processing: Implications for psychology and neurobiology (pp. 131-181). Oxford: Oxford University Press, Clarendon Press.

Rayner, K., Murphy, L. A., Henderson, J. M., \& Pollatsek, A. (1989). Selective attentional dyslexia. Cognitive Neuropsychology, 6, 357-378.

Rayner, K., Pollatsek, A., \& Bilsky, A. B. (1995). Can a temporal processing deficit account for dyslexia? Psychonomic Bulletin \& Review, 2, 501-507.

REED, M. A. (1989). Speech perception and the discrimination of brief auditory cues in reading disabled children. Journal of Experimental Child Psychology, 48, 270-292.

Robertson, L., Knight, R., Rafal, R., \& Shimamura, A. (1993). 
Cognitive neuropsychology is more than single-case studies. Journal of Experimental Psychology: Learning, Memory, \& Cognition, 19, 710-717.

Seidenberg, M., \& McClelland, M. (1989). A distributed developmental model of word recognition and naming. Psychological Review, 96, 523-568.

Seymour, P. H. K., \& MacGregor, C. J. (1984). Developmental dyslexia: A cognitive experimental analysis of phonological, morphemic, and visual impairments. Cognitive Neuropsychology, 1, 43-83.

ShalliCE, T. (1988). From neuropsychology to mental structure. New York: Cambridge University Press.

Shallice, T., \& Warrington, E. K. (1977). The possible role of selective attention in acquired dyslexia. Neuropsychologia, 15, 31-41.

Shapiro, K. L., Ogden, N., \& Lind-Blad, F. (1990). Temporal processing in dyslexia. Journal of Learning Disabilities, 23, 99-107.

SNowling, M., \& Hulme, C. (1989). A longitudinal case study of developmental phonological dyslexia. Cognitive Neuropsychology, 6, 379-401.

Snowling, M., Stackhouse, J., \& Rack, J. (1986). Phonological dyslexia and dysgraphia-a developmental analysis. Cognitive Neuropsychology, 3, 309-339.

STANOVICH, K. E. (1988). Explaining the differences between the dyslexic and the garden-variety poor reader: The phonological-core variable-difference model. Journal of Learning Disabilities, 21, 590-604.

Stothard, S., Snowling, M., \& Hulme, C. (in press). Deficits in phonology but not dyslexic? Cognitive Neuropsychology.

STUdDERT-KenNedY, M., \& MODY, M. (1995). Auditory temporal perception deficits in the reading-impaired: A critical review of the evidence. Psychonomic Bulletin \& Review, 2, 508-514.

TAllal, P. (1980). Auditory temporal perception, phonics, and reading disabilities in children. Brain \& Language, 9, 182-198.

Temple, C. M., Jeeves, M. A., \& Vilarroya, O. O. (1990). Reading in callosal agenesis. Brain \& Language, 39, 235-253.

Temple, C. M., \& Marshall, J. (1983). A case study of developmental phonological dyslexia. British Journal of Psychology, 74, 517-533.

Van Orden, G. C., Pennington, B. F., \& Stone, G. O. (1990). Word identification in reading and the promise of subsymbolic psycholinguistics. Psychological Review, 97, 488-522.

ZURIF, E., Gardner, H., \& BRownell, H. (1989). The case against the case against group studies. Brain \& Cognition, 10, 237-255.

\section{NOTES}

1. Although there are defenders of the group study approach to adult neuropsychology (e.g., Robertson, Knight, Rafal, \& Shimamura, 1993; Zurif, Gardner, \& Brownell, 1989), the pitfalls of the approach appear to be well established (see McCloskey, 1993, for a response to Robertson et al., 1993). For illustrative examples of the problems arising from group research, see the discussion on amnesia research by Shallice (1988, pp. 25-29) and the discussion on agrammatism by Ellis and Young (1988, pp. 241-251).

2. A current debate in the neuropsychological literature is whether a "third route" for oral reading must be postulated in which whole-word orthographic representations are mapped directly onto whole-word phonological representations, bypassing semantics. See Goodall and Phillips (1995) and Hillis and Caramazza (1991) for contrasting views.

3. Connectionist models have recently been proposed which model the reading of regular and irregular words and nonwords through a single route (Seidenberg \& McClelland, 1989; Van Orden, Pennington, \& Stone, 1990). Although an attempt has been made to mimic some of the data from acquired dyslexic subjects through "lesioning" of the connections or nodes in these models (e.g., Patterson, 1990; Patterson, Seidenberg, \& McClelland, 1989), the success of these attempts is open to debate. According to Coltheart, Curtis, Atkins, and Haller (1993), the dual-route approach readily accommodates the data from acquired dyslexia, whereas the single-route approach has yet to provide a compelling account.

4. Difficulty in recognizing whole words is often attributed to a visual deficit. However, in order to carry out grapheme-phoneme conversion, and thereby read nonwords and regular words correctly, it is necessary to recognize individual letters. Thus, some explanation is needed for why there is a deficit in recognizing larger groups of letters but not individual letters. Also, describing the impaired process as visual seems somewhat misleading, since what is needed is orthographic recognition-that is, recognizing many different visual forms (such as uppercase, lowercase, cursive) as representations of the same orthographic units.

5. Of course, even if a significant correlation is obtained, one is left with explaining why there are exceptions-that is, individuals with temporal processing deficits but no phonological deficits or the reverse. An example of such a dissociation was reported in Ludlow, $\mathrm{Cu}-$ dahy, Bassich, and Brown (1983). This study demonstrated an auditory temporal discrimination impairment in children with attention deficit disorder (ADD) but no reading deficit. Although it might seem plausible that this temporal processing disorder resulted from the subjects' attentional deficit, Ludlow et al. took some pains to demonstrate that the deficit could not be attributed to failure to attend to the task. For example, they showed that there was no correlation between the ADD children's performance on a vigilance task and their performance on the temporal discrimination task.

(Manuscript received May 16, 1995; revision accepted for publication July 11, 1995.) 